\title{
The tango of ROS and p53 in tissue stem cells
}

\author{
Youguo Chen ${ }^{1} \cdot$ Keli $\mathrm{Liu}^{2} \cdot$ Yufang $\mathrm{Shi}^{1,2} \cdot$ Changshun Shao $\mathbb{( D )}^{1}$
}

Received: 2 January 2018 / Accepted: 3 January 2018 / Published online: 27 February 2018

(c) The Author(s) 2018

The $\mathrm{p} 53$ protein is a master regulator of cellular response to many types of stress, including oxidative stress. Depending upon the cellular context and the extent to which p53 is activated, this protein is capable of initiating programs that can eventually lead to inhibition of cell proliferation, induction of cell differentiation, modulation of metabolism, and induction of apoptosis or cellular senescence [1]. The functional versatility and complexity of p53 are mediated by the hundreds of p53 transcriptional targets. Reactive oxygen species (ROS) are essential signaling molecules in many biological processes, wherein their level is tightly regulated. Excess ROS may cause apoptosis, premature senescence or unscheduled differentiation of stem cells. Various studies showed that, under physiological conditions or when exposed to transient and mild oxidative stress, p53 possesses antioxidant function and contributes to the maintenance of low level of ROS [2]. The list of p53 target genes that encode proteins with antioxidant function has been growing over the years and now includes GPX1, SOD2, TIGAR, SESN1, SESN2,GLS2 and SLC2A9. However, the antioxidant function of p53 is only context-dependent. When under persistent and severe stress, p53 becomes hyperactivated and turns into a strong promoter of oxidative stress instead. This pro-oxidative activity of p53 is believed to be driven by its transcriptional activation of genes encoding pro-oxidative proteins such as TP53I3 (PIG3) and excessive ROS level often lead to apoptosis or cellular senescence [3-5]. Because p53 can be further activated or maintained at high levels by persistent high level of ROS,

Changshun Shao

shaoc@suda.edu.cn

1 The First Affiliated Hospital of Soochow University, State Key Laboratory of Radiation Medicine and Radioprotection, Institutes for Translational Medicine, Soochow University, Suzhou, Jiangsu 215123, China

2 Institute of Health Sciences, Shanghai Institutes for Biological Sciences of Chinese Academy of Sciences and Shanghai Jiao Tong University School of Medicine, Shanghai 200025, China the p53 and ROS eventually form a positive feedback loop, resulting in a vicious cycle and further exacerbating oxidative stress $[5,6]$. While the antioxidant function of p53 is mainly attained via upregulating the classical antioxidant enzymes, the mechanisms by which p53 elevates ROS are less understood. Nevertheless, p21, a classical p53 target, has been shown to have pro-oxidant function [5]. A recent study by Kang et al showed that p53 and PIG3 can each interact with and inhibit catalase when p53 is hyperactivated, leading to high ROS level and apoptosis [7]. Interestingly, the study also showed that p53R2, another p53 target gene, actually contributes to the maintenance of increased catalase activity under physiological conditions. How p53 is shifted in its function from being an antioxidant to a pro-oxidant under different stress levels remains to be elucidated.

Proper regulation of ROS homeostasis plays an essential role in fate decision of tissue stem cells. Mesenchymal stem cells (MSCs) are ubiquitous in mammals and can give rise to osteoblasts, chondrocytes and adiopocytes. An early study showed that excessive p53 activity blocks osteoblast differentiation and p53 deficiency results in increased osteogenesis [8]. However, p53 was later shown to also restrict white adipogenic differentiation and protect against diet-induced obesity [9]. Interestingly, unlike the inhibitory effect it has on white adipocyte differentiation, p53 positively regulates brown adipocyte differentiation, possibly via upregulating PRDM16, a transcription factor required for brown fate lineage development. Adipocyte differentiation has long been known to be characterized by increased production of ROS. Tormos et al. demonstrated that ROS generation by mitochondria is required for adipocyte differentiation of primary human MSCs [10]. Particularly, ROS are generated from mitochondrial complex III in an mTORC1 signaling-dependent manner. These results clearly show that mitochondrial metabolism and ROS generation drive adipocyte differentiation, instead of being its consequences. A study reported in this issue of CDD provides further support that the p53-ROS axis plays an essential role in adipocyte differentiation [11]. The authors 
Fig. 1 ROS and p53 determine the differentiation routes of MSCs. Hyperactivation of p53 drives mitochondrial ROS production and adipocyte differentiation. p53 functions as an antioxidant under physiological condition or mild stress. MSCs are favored to differentiate into osteoblasts when p53 is absent or functionally compromised

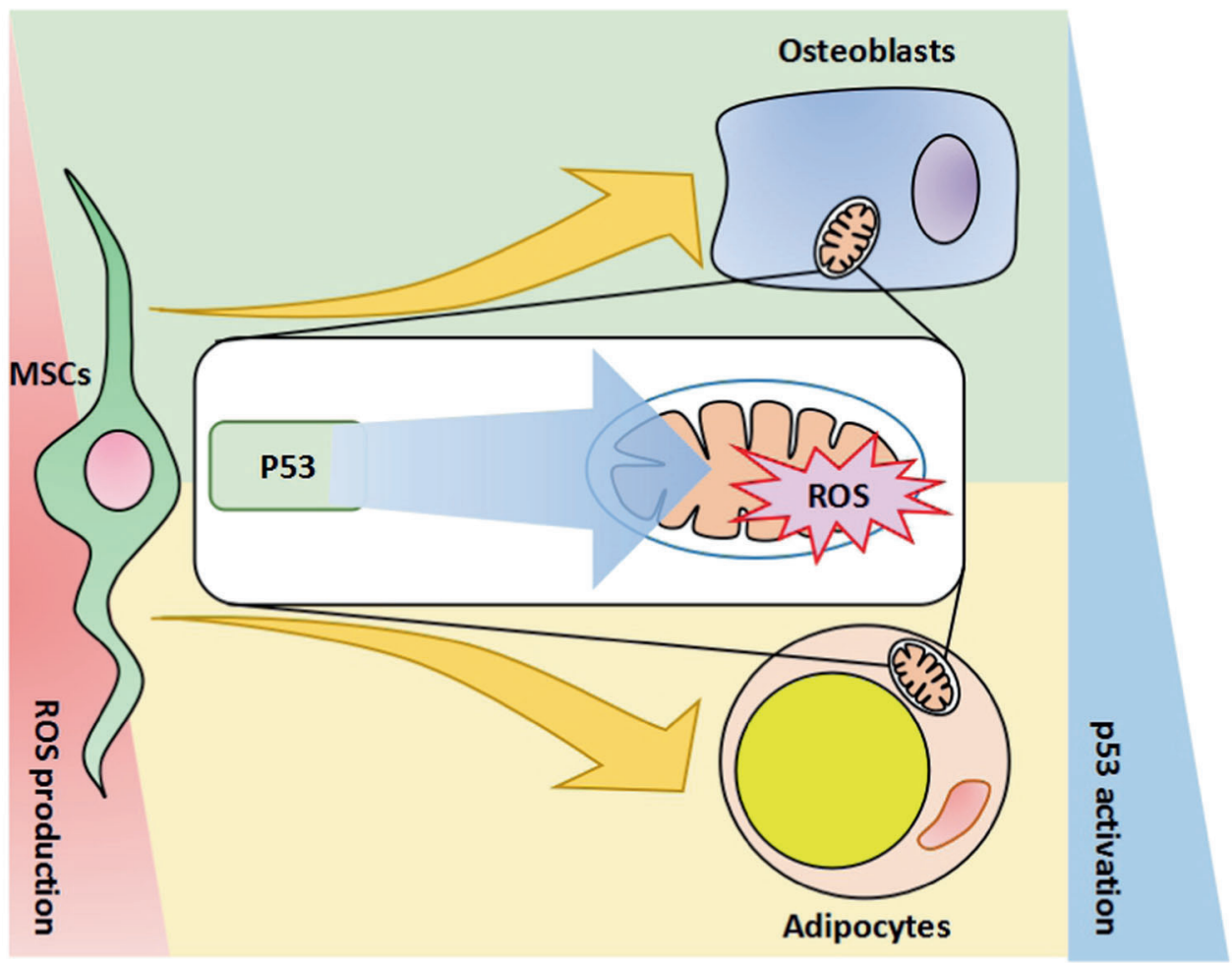

showed that, in murine MSCs, lack of p53 could promote osteogenesis at the expense of adipogenesis. Interestingly, p53 function is also required for the production of mitochondrial superoxide that drives adipogeneic differentiation. Diminished mitochondrial ROS production in p53-null MSCs led to impaired adipogenesis. Osteogenesis, on the other hand, was favored when ROS production was attenuated. Thus, it appears that a p53-ROS axis positively regulates adipogenesis while inhibiting osteogenesis (Fig. 1). However, it remains unclear how p53 promotes the production of mitochondrial ROS in MSCs.

ROS and p53 are also critically involved in the differentiation of neural progenitor cells (NPCs). During prenatal development, lack of p53 in NPCs leads to elevated ROS and premature neuronal differentiation, which can be partially rescued by ectopic expression of p53 or antioxidant treatment [12]. However, NPCs are not affected in their proliferation and astrocytic differentiation in the absence of p53. Sesn2, an antioxidant encoded by p53, contributes to the reduction of ROS in NPCs. These results suggest that p53 fine-tunes endogenous ROS levels to ensure the appropriate timing of prenatal neurogenesis. In contrast, p53 appears to play a different role in postnatal neural stem cells (NSCs). Deletion of FIP200, which is essential for autophagy induction, resulted in a progressive loss of NSCs and an impairment in neuronal differentiation in the postnatal brain [13]. The apoptotic responses and cell cycle arrest accounting for the postnatal loss of NSCs were p53dependent. However, the impaired neuronal differentiation was independent of p53, and but could be rescued by antioxidant $\mathrm{N}$-acetylcysteine. These studies suggest that ROS regulation in NPCs/NSCs by p53 operates in a developmental stage specific manner.

The tango of ROS and p53 could drive the depletion of hematopoietic stem cells (HSCs) [14]. ROS levels were elevated and thus activated $\mathrm{p} 53$ in the bone marrow of Mdm2 null and p53 hypomorphic mice. In the absence of Mdm2, the stable p53 further induced ROS and caused cell cycle arrest, senescence and apoptosis of HSCs and other hematopoietic cells [14]. However, p53 was also found to maintain the pool of HSCs by reducing ROS level under a different condition [15]. Thioredoxin-interacting protein (TXNIP) was shown to upregulate p53 by interfering with MDM2-p53 interactions, and thus to increase the transcription of antioxidant genes. Txnip(-/-) mice showed a downregulation of antioxidant genes. Introduction of TXNIP or p53 into Txnip $(-1-)$ bone marrow cells rescued the HSC frequency. These results indicate that whether p53 functions to elevate or to reduce ROS in HSCs is highly context-dependent.

Redox homeostasis is essential for the healthy selfrenewal, maintenance of quiescence and proper differentiation of tissue stem cells. It appears that p53 regulates these processes by acting as either an antioxidant or a pro-oxidant. On the other hand, oxidative stress caused by excessive production of ROS or by defective antioxidant system also exerts its deleterious effects via hyperactivation of $\mathrm{p} 53$. As various tissue stem cells are being further explored, we are expected to learn many new acts of p53 and ROS. 
Acknowledgements We are supported by National Natural Science Foundation grants 81572785 and 81530043 .

\section{Compliance with ethical standards}

Conflict of interest The authors declare that they have no conflict of interest.

Open Access This article is licensed under a Creative Commons Attribution-NonCommercial-ShareAlike 4.0 International License, which permits any non-commercial use, sharing, adaptation, distribution and reproduction in any medium or format, as long as you give appropriate credit to the original author(s) and the source, provide a link to the Creative Commons license, and indicate if changes were made. If you remix, transform, or build upon this article or a part thereof, you must distribute your contributions under the same license as the original. The images or other third party material in this article are included in the article's Creative Commons license, unless indicated otherwise in a credit line to the material. If material is not included in the article's Creative Commons license and your intended use is not permitted by statutory regulation or exceeds the permitted use, you will need to obtain permission directly from the copyright holder. To view a copy of this license, visit http://creativecommons. org/licenses/by-nc-sa/4.0/.

\section{References}

1. Kastenhuber ER, et al. Cell. 2017;170:1062-78.

2. Liu B, et al. Free Radic Biol Med. 2008;44:1529-35.

3. Johnson TM, et al. Proc Natl Acad Sci USA. 1996;93:11848-52.

4. Polyak K, et al. Nature. 1997;389:300-5.

5. Passos JF, et al. Mol Syst Biol. 2010;6:347.

6. Wei Z, et al. Free Radic Biol Med. 2015;79:1-13.

7. Kang MY, et al. Cell Death Differ. 2013;20:117-29.

8. Lengner CJ, et al. J Cell Biol. 2006;172:909-21.

9. Molchadsky A1, et al. Cell Death Differ. 2013;20:774-83.

10. Tormos KV, et al. Cell Metab. 2011;14:537-44.

11. Krishnappa V, et al, Cell Death Differ. https://doi.org/10.1038/ s41418-017-0004-4.

12. Forsberg K, et al. J Neurosci. 2013;33:14318-30.

13. Wang C, et al. Nat Neurosci. 2013;16:532-42.

14. Abbas HA, et al. Cell Stem Cell. 2010;7:606-17.

15. Jung H, et al. Cell Metab. 2013;18:75-85. 